\title{
EEG signal based Modified Kohonen Neural Networks for Classification of Human Mental Emotions
}

\author{
D. Jude Hemanth ${ }^{1}{ }^{*}$ \\ ${ }^{1}$ Department of ECE, Karunya Institute of Technology and Sciences, Coimbatore, India \\ *Corresponding Author: D. Jude Hemanth, Email: judehemanth@karunya.edu
}

\begin{abstract}
How to cite this paper: D. Jude Hemanth (2020). EEG signal based Modified Kohonen Neural Networks for Classification of Human Mental Emotions. Journal of Artificial Intelligence and Systems, 2, 1-13. https://doi.org/10.33969/AIS.2020.21001.

Received: December 11, 2019

Accepted: January 17, 2020

Published: January 23, 2020

Copyright $($ C 2020 by author(s) and Institute of Electronics and Computer.

This work is licensed under the Creative Commons Attribution International License (CC BY 4.0).

http://creativecommons.org/licenses/by/4.0/

(c) (i) Open Access
\end{abstract}

\begin{abstract}
Identifying human emotions is very important in human machine interaction(HMI). These emotions will affect communication between people, and their mood.Emotion detection will give a clear idea aboutcustomer satisfaction in e-learning, marketing, entertainments and behavior of criminals in law. Artificial neural networksare essential for machine learning and emotion detection.The emotions are detected from EEG signals which can give better performance to audio and facial signals. In this work, several modified Kohonen neural networks are proposed for human emotion classification.EEG signals from DEAP Database are used asinput for ANN to detect the human emotions. Angry, Happy, Sad and Relax are the emotions classified using KohonenNeural Networks.Experimental results show promising results for the proposed approaches.
\end{abstract}

Keywords

Kohonen neural network, brain signals, human emotions, classification accuracy

\section{Introduction}

Human emotion recognition will improve the effective communication between human and machine interaction. Multi-model bio signals are used to classify the human emotions. Six human emotions are classified using back propagation artificial neural network. Accuracy of this model is good [1]. Emotions are recognized using physiological-data-driven approach by Multiple-fusion-layer based ensemble classifier of stacked autoencoder (MESAE).Three hidden layers and one filter is present in SAE to avoid the noise and stable features. DEAP dataset is given as an 
input to this classifier. Shallow emotion classifier and state-of-the-art deep learning models has lower generalization capability than MESAE [2].Emotion recognition using EEG signals is difficult but in Deep Neural Network (DNN), EEG-ER is more accurate than human's recognition capability. DEAP EEG data signal is given as an input to the DNN model. This will classify the emotion in two levels "positive" and the other one is "negative". Finally, CNN and conventional models are compared [3].

Affective states classification problem is addressed in two types Stacked denoising autoencoder (SDAE) and Deep Belief Networks (DBN). DEAP dataset EEG signals are given as an input to this model. In DPN 86.67\%, 86.60\% and 86.69\% are the average F1 scores of arousals, valence and liking states. Small fraction of labeled data is applied for semi-supervised learning models. It will increase the state-of-the-art classification performance [4]. Here, emotions are identified using facial and brain signals. Feature extraction of both signals are calculated and given to artificial neural network for emotion recognition. Active Shape Model is used for facial feature extraction and Support Vector Machine method is used for emotion classification [5].

DEAP data set EEG brain signals features are extracted from Shannon Entropy and higher order auto-regressive model of statistical measures. These features are given as an input to multi-class Support Vector Machine for emotion classification. Accuracy of this algorithm is $94.097 \%$ [6]. Brain will produce different signals for every emotion. These changes are observed to reduce the noise and artifacts in EEG signal, modified adaptive filtering method is used. Feature extraction values of EEG signal is given to the adaptive neuro fuzzy inference system for emotion classification and analysis [7]. Facial expression, gesture, speech and text are the different ways to find the human emotions. Six emotions are classified by 4 classification methods SVM, NN, KNN, Naive Bayes and DWT. In neural network method 55.58\% of accuracy is measured [8]. Feature extraction of EEG signal is calculated using discrete wavelet transforms. Extracted features are given to support vector machine and K-nearest neighbor classifiers to detect the emotional states. Arousal has the maximum accuracy of $86.75 \%$ and the valence accuracy of $84.05 \%$ [9].Feature extraction, dynamical reduction is calculated using Principle Component Analysis and Wavelet Transform. In accuracy Wavelet transform with ANN is having less accuracy than PCA with ANN. Various optimization techniques are used to improve the accuracy in Artificial Neural Network [10].

EEG signal frequency range is divided into five different levels according to their power spectrum density. 0 to $4 \mathrm{~Hz}$ EEG low frequency signals are avoided to reduce the EEG artifacts. Human emotions are detected by using inference and Bayesian network [11]. Real time EEG signals are recorded by Ground Truth Method. Extracted features are given to k-NN classifier then both sad and happy signals are 
classified. Training and testing data of different combinations are given to the classifier to calculate the accuracy level [12]. Recognizing functional autism patient emotion is very difficult using facial expression. Deep learning algorithm is used for both feature extraction and emotional classification. Three layers of restricted Boltzmann machines problems are cleared by Deep learning algorithm. Comparing to conventional algorithm, Deep learning algorithm has better emotion recognition accuracy [13].

In brain electrical activity of neurons are recorded by EEG. Support vector Machine (SVM), Linear Discriminant (LDA) classifiers are used to classify seven human emotions. Accuracy of both classifiers is $74.13 \%$ and $66.50 \%$ respectively. If the training sample increases it will increase the accuracy. While comparing both classifiers SVM has given a good accuracy of emotion recognition [14]. From EEG signals, sub-signals are plotted with the help of 3-D phase space diagram. From this diagram mean, standard deviation of Euclidian distance is calculated. Human emotions are classified with the help of multiclass least squares support vector machines (MC-LS-SVM).This classifier has an accuracy of 91.04\% [15].

Three human emotions are classified with the help of hierarchical network structure with subnetwork nodes. Two EEG signal database is given to this system with both single and multiple modality. This classifier is compared with other state-of-the-art methods and shows that it performs better than them [16]. EEG signals are recorded from the object while they are watching a music video. This is given to the probabilistic network, which is simple, efficient, and easy to train. In order to reduce the number of channels Relief-based channel selection algorithm is used. Reducing the number of channels will not affect the accuracy of the system [17]. A simple Deep Neural network and convolutional neural networks are used for classification of emotions using DEAP EEG brain signals. This model has a state-of-the-art classification accuracy of 4.51 [18]. But, this method is computationally complex. Hierarchical convolutional Neural network is used to classify positive, neutral and negative emotions of EEG signals. And also stacked autoencoder (SAE), SVM and KNN classifiers also used for classification purpose. But accuracy of HCNN is higher than SAE. High frequency wave bands of EEG signals, Beta and Gamma waves used to read the emotions [19]. EEG signals of SEED dataset (SJTU Emotion Dataset) are used to find the time domain and time-frequency domain features. Artificial Neural network and Support Vector Machine (SVM) classifiers are used to classify three different human emotions with the accuracy rate of 91.2\% [20]. But. Several methods have given better accuracy.

Training algorithm of Levenberg-Marquardt is used in the feed-forward neural network. Emotion-specific multichannel EEG dataset are given as input to this classifier. It has $60 \%$ of accuracy in emotion recognition [21].BDDAE and Bimodal-LSTM models are called denoising autoencoders. Features of temporal 
information and frequency-domain information are used in this classifier. Both SEED and DEAP data sets are given to this system. The mean accuracy is measured as $93.97 \%$ and $83.53 \%$ respectively [22]. When the music is played, EEG brain signals are categorized by self-reported emotional states. With the help of support vector machine classifier four emotions (joy, anger, sadness, and pleasure) are classified with the average accuracy of $82.29 \%$ [23]. Russell's circumplex model, Higuchi Fractal Dimension (HFD) and support machine Vector are used to classify the emotions. Machine learning is done in two ways, one is for all subjects and another one is for a particular subject. In this first method is impossible for real time application and the second one is suitable for real time application and it has $70.5 \%$ of accuracy[24].In Support Vector Machine classifier emotion recognition is done based on Thayer's two dimensional model and accuracy of the classifier is increased from $72.14 \%$ to $87.27 \%$ with hierarchical classifier[25]. The novelty and the contributions of this work are:

- Two modified Kohonen neural networks are proposed in this work.

- The modifications are performed in the training algorithm. Specifically, the changes are made in the weight adjustment process of the conventional system.

- The proposed modified neural networks are independent of the difference between the inputs and old weights unlike the conventional Kohonen neural networks. This change has improved the performance of the proposed system

Since the current society is suffering from stress, depression, anxiety, the proposed work is highly suitable for practical applications. Smart watches can be developed with the proposed works as computational algorithms. People can use them daily to assess their emotional situation in workplaces. Similarly, phycologists can use these algorithms in their computers to assess the emotional conditions of their patients. 


\section{Proposed approach}

The proposed approach is shown in Figure 1.

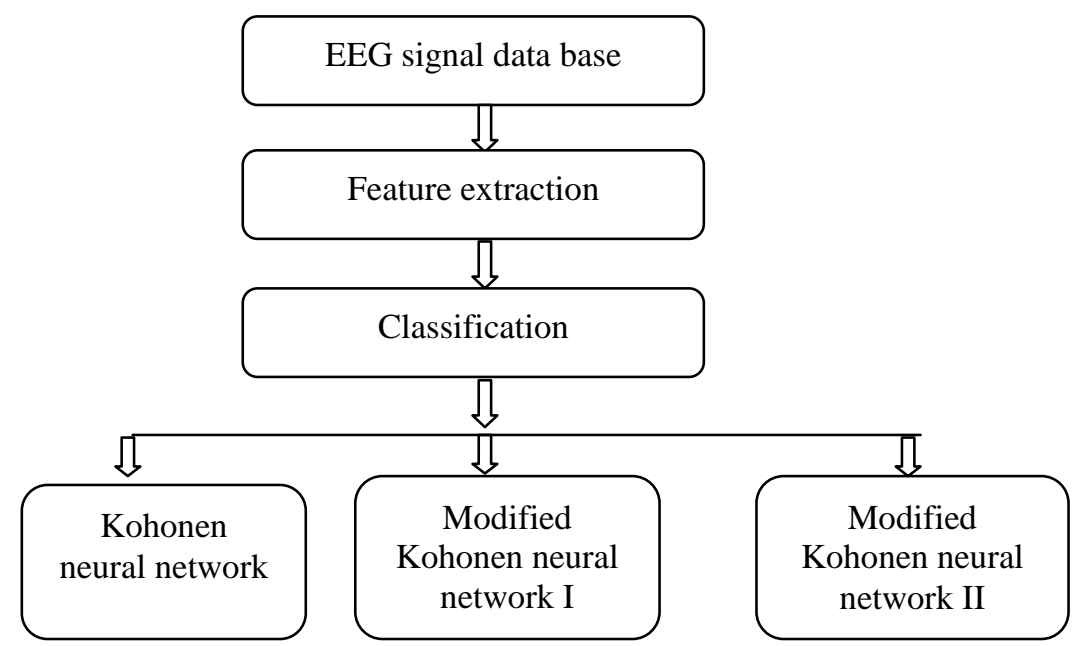

Figure 1. Framework of the proposed approach.

EEG brain signal will give an accurate emotion while comparing to facial,speech,gesture signals. Feature extraction of these signals is tabulated. Kohonen neural network is used to classify the human emotions. In order to improve the accuracy some modification is made in Kohonen neural network.

\subsection{EEG signal database}

Emotion recognition of human is more accurate when we use EEG brain signal because participants can hide their feeling in speech and facial signals. But it will not happen in brain signal. Here DEAP dataset [26] is used for emotion classification. In this dataset 32 participants are asked to watch a 40, one minute videos and their EEG brain signals are recorded in a noise free environment. Arousal and valences are responsible for classification of human emotion. If both the values are high it will be happy or relax, if both the values are low it will be angry or sad.

\subsection{Feature extraction}

Feature extraction is used to extract the information from the signals. Mean, Variance, standard deviation, skewness, kurtosis, mobility and complexity are the features taken to analysis the EEG brain signal.

\section{Mean:}

Mean value of the entire EEG signal is measured by the given formula. 


$$
\text { Mean }=\frac{1}{N} \sum_{i-1}^{N} X_{i}
$$

Variance:

Variance is also one parameter in the feature of the signal. This is calculated by the given formula

$$
\text { Variance }=\sigma^{2}
$$

Median:

Median value of a EEG signal is tabulated using the below equation

$$
\left\{\begin{array}{cc}
Y_{\left(N+\frac{1}{2}\right)} & \text { if } N \text { is odd } \\
1(Y(N / 2)+Y 2+N / 2) & \text { if } N \text { is even }
\end{array}\right\}
$$

Where Y1 and Y2 are two different EEG signals.

\section{Mobility:}

Ratio between first derivative of variance and variance is measured as mobility of the signal.

$$
\text { Mobility }=\frac{\text { First derivative of Varience }}{\text { Varience }}
$$

\section{Complexity:}

Ratio between first derivative of mobility and mobility is called complexity of the EEG signal.

$$
\text { Complexity }=\frac{\text { First derivative of Mobiligy }}{\text { Mobility }}
$$

\section{Skewness:}

Symmetry of the EEG signal is calculated using skewness formula. It always depends on mean and variance of the EEG signal.

$$
\text { Skewness }=\frac{E(\mathrm{x}-\mu)^{3}}{\sigma^{3}}
$$

\section{Kurtosis:}

Kurtosis of the signal gives the information about the peaks present the EEG signals. This is measured by the given formula where ' $m$ ' corresponds to the moments. 


$$
\text { Kurtosis }=\frac{m_{4}}{m_{2}^{2}}
$$

\section{Classification}

Human emotions are classified using Kohonen neural network. Angry, happy, relax and sad are the four human emotions classified using valence an arousal value. If both valence and arousal values are high then it will be a positive emotion happy, relax. If both are low then it will be a negative emotion angry or sad.

\subsection{Kohonen neural network}

Kohonen neural network is also called self-organized map. It is a single layer, unsupervised, feed forward artificial neural network. Only one hidden layer is present in the Kohonen neural network. It uses “winner take -all” training rule for the training algorithm. Complexity of this network is less in comparison to Back Propagation Neural Network.

\section{Training algorithm of Kohonen neural network:}

Step1: Supply first input(x), randomly initialized weight matrix (w), learning $\operatorname{rate}(\alpha)$.

Step2: Determine the Euclidean distance for every output layer.

$$
d(j)=\sum_{i \neq 1}^{N}\left(x_{i}-w_{\text {old }}\right)^{2}
$$

Step3: Determine the neuron (j) for which the distance value is minimum.

Step4: Adjust only the weights of the winner neuron

$$
w_{i j}(\text { new })=w_{i j}(\text { old })+\propto\left[\mathrm{x}_{i}-w_{i j} \text { (old) }\right]
$$

Step5: Repeat step 2 to step 4 with the new set of weights.

Step6: Repeat the algorithm for a specified number of iterations (100 to 200 times).

\subsection{Modified Kohonen neural network I}

In Kohonen neural network is an unsupervised network, so accuracy level is low while comparing to BPN. In order to increase the accuracy level some modification is introduced in the training algorithm. In Kohonen neural network new weight value is strong dependence of the initialization. So, in this modified Kohonen neural network new weight value will not dependent on the previous weight values.

\section{Training algorithm of modified kohonen I:}

Step1: Supply first input(x), randomly initialized weight matrix(w),learning rate( $\alpha$ ). Step2: Determine the Euclidean distance for every output layer. 


$$
d(j)=\sum_{i \neq 1}^{N}\left(\mathrm{x}_{i}-w_{\text {old }}\right)^{2}
$$

Step 3: Determine the neuron (j) for which the distance value is minimum.

Step4: Adjust only the weights of the winner neuron

$$
\Delta w_{i j}=2 \propto x_{i}+\propto
$$

Step5: Repeat step 2 to step 4 with the new set of weights.

Step6: Repeat the algorithm for a specified number of iteration.(150).

The value of alpha used in this work is 0.6. The weight adjustment process is different from the conventional system. In the proposed method, the weight adjustment process is independent of the difference between the inputs and the old weights. Since this difference can never be zero, the conventional system performance is relatively poor. This is overcome in the proposed method.

\subsection{Modified Kohonen neural network II}

In modified kohonen neural network II, few other changes are made to increase the accuracy level in comparison to normal Kohonen neural network. Accuracy level of this network is higher than normal Kohonen and lesser than modified Kohonen neural network II.

\section{Training algorithm of modified Kohonen II:}

Step1: Supply first input(x), randomly initialized weight matrix(w), learning rate( $\alpha$ ). Step2: Determine the Euclidean distance for every output layer.

$$
d(j)=\sum_{i \neq 1}^{N}\left(\mathrm{x}_{i}-w_{\text {old }}\right)^{2}
$$

Step 3: Determine the neuron ( $\mathrm{j}$ ) for which the distance value is minimum.

Step4: Adjust only the weights of the winner neuron

$$
\Delta W_{i j}=2 \propto x_{i}-\propto^{2}
$$

Step5: Repeat step 2 to step 4 with the new set of weights.

Step6: Repeat the algorithm for a specified number of iterations. (150)

The value of alpha used in this work is 0.6

\section{Experimental Results and Discussions}

The tests are completed in an Intel Core(TM) i3 processor with 4 GB RAM. The product utilized for the execution is MATLAB. Four human emotions of angry, happy, relax and sad are classified using Kohonen neural network.384 EEG signals are taken as an input to this test. There are 96 signals in each category. Among these, 30 signals are used for training process and the remaining 66 signals are used for testing process 


\subsection{Classification Accuracy of the classifiers}

The proposed classifiers are analyzed by the terms accuracy, sensitivity and specificity. Formulae for measuring these terms are given below:

$$
\begin{aligned}
& \text { Classification } \operatorname{Accuracy}(C A)=\frac{T P+T N}{(T P+T N+F P+F N)} \\
& \text { Sensitivity }(S N)=\frac{T P}{(T P+F N)} \\
& \text { Sensitivity }(S P)=\frac{T N}{(T N+F P)}
\end{aligned}
$$

Where $\mathrm{TP}=$ True Positive, $\mathrm{TN}=$ True Negative, $\mathrm{FP}=$ False Positive, $\mathrm{FN}=\mathrm{False}$ Negative. The detailed information about the performance measures and TP, FP, FN and TN are available in [27]. More information is available in [28-34].

The confusion matrix values are shown in Table 1, 2 and 3

Table 1. Confusion Matrix of Normal Kohonen network

\begin{tabular}{|c|c|c|c|c|}
\hline & ANGRY & HAPPY & RELAX & SAD \\
\hline ANGRY & 40 & 0 & 0 & 26 \\
\hline HAPPY & 0 & 47 & 0 & 19 \\
\hline RELAX & 0 & 17 & 26 & 23 \\
\hline SAD & 0 & 0 & 21 & 45 \\
\hline
\end{tabular}

Table 2. Confusion Matrix of Modified Kohonen network I

\begin{tabular}{|c|c|c|c|c|}
\hline & ANGRY & HAPPY & RELAX & SAD \\
\hline ANGRY & 40 & 0 & 0 & 26 \\
\hline HAPPY & 0 & 47 & 0 & 19 \\
\hline RELAX & 0 & 8 & 34 & 24 \\
\hline SAD & 0 & 0 & 21 & 45 \\
\hline
\end{tabular}

Table 3. Confusion Matrix of Modified Kohonen network II

\begin{tabular}{|c|c|c|c|c|}
\hline & ANGRY & HAPPY & RELAX & SAD \\
\hline ANGRY & 40 & 0 & 0 & 26 \\
\hline HAPPY & 0 & 47 & 0 & 19 \\
\hline RELAX & 0 & 16 & 27 & 23 \\
\hline SAD & 0 & 0 & 21 & 45 \\
\hline
\end{tabular}

From the above table, it is evident that the misclassification of emotion is less in modified Kohonen neural network and more in normal Kohonen neural network. Then accuracy, sensitivity and specificity values are calculated using above formulae with the help of confusion matrix. These values are shown in Table 4,5 and 6. 
Table 4. Performance measures of Normal Kohenen neural network

\begin{tabular}{|c|c|c|c|c|c|c|c|}
\hline & $\mathrm{TP}$ & TN & FP & FN & CA & SN & SP \\
\hline ANGRY & 40 & 198 & 0 & 26 & 0.9015 & 0.6060 & 1 \\
\hline HAPPY & 47 & 181 & 17 & 19 & 0.8636 & 0.7121 & 0.9141 \\
\hline RELAX & 26 & 177 & 21 & 40 & 0.7689 & 0.3939 & 0.8939 \\
\hline SAD & 45 & 130 & 68 & 21 & 0.6628 & 0.6818 & 0.6565 \\
\hline \multicolumn{5}{|c|}{ verage values } & 0.7992 & 0.5984 & 0.8661 \\
\hline
\end{tabular}

Table 5. Performance measures of Modified Kohonen network I

\begin{tabular}{|c|c|c|c|c|c|c|c|}
\hline & $\mathrm{TP}$ & TN & FP & FN & CA & SN & SP \\
\hline ANGRY & 40 & 198 & 0 & 26 & 0.9015 & 0.6060 & 1 \\
\hline HAPPY & 47 & 190 & 8 & 19 & 0.8977 & 0.7121 & 0.9595 \\
\hline RELAX & 34 & 177 & 21 & 32 & 0.7992 & 0.5151 & 0.8939 \\
\hline SAD & 45 & 129 & 69 & 21 & 0.6590 & 0.6818 & 0.6515 \\
\hline \multicolumn{5}{|c|}{ verage values } & 0.8143 & 0.6287 & 0.8762 \\
\hline
\end{tabular}

Table 6. Performance measures of Modified Kohonen network II

\begin{tabular}{|c|c|c|c|c|c|c|c|}
\hline & TP & TN & FP & FN & CA & SN & SP \\
\hline ANGRY & 40 & 198 & 0 & 26 & 0.9015 & 0.6060 & 1 \\
\hline HAPPY & 47 & 182 & 16 & 19 & 0.8674 & 0.7121 & 0.9191 \\
\hline RELAX & 27 & 177 & 21 & 39 & 0.7727 & 0.4090 & 0.8939 \\
\hline SAD & 45 & 130 & 68 & 21 & 0.6628 & 0.6818 & 0.6565 \\
\hline \multicolumn{5}{|c|}{ Average values } & 0.8011 & 0.6022 & 0.8673 \\
\hline
\end{tabular}

The Kohonen neural networks have high specificity values than sensitivity values. The proposed method of neural networks has high accuracy in comparison to normal Kohonen neural networks. It may be noted that the modified Kohonen networks will not depend on the old weight values. A comparative analysis with other works is given in Table 7.

Table 7. Comparative analysis with other works

\begin{tabular}{|c|c|c|}
\hline Author & Method & Accuracy (\%) \\
\hline \multirow{3}{*}{ Youjun Li et al [28] } & K-NN & 46 \\
\cline { 2 - 3 } & Random decision forest & 39 \\
\cline { 2 - 3 } & SVM & 65 \\
\cline { 2 - 3 } & CNN+RNN & 61 \\
\hline D. Jude Hemanth & Conventional Kohonen network & 86 \\
\hline D. Jude Hemanth & Modified neural network I & 87 \\
\hline D. Jude Hemanth & Modified neural network II & 86 \\
\hline
\end{tabular}

It is evident from the table that the proposed methods perform in an efficient way for practical applications. 


\section{Conclusions}

Modified Kohonen neural networks are proposed in this work to improve the accuracy level of human emotion classification process. An improvement of 1-2\% is achieved in modified Kohonen neural networks I and II. Even though the value is marginal, it is highly significant in case of affective computing applications. As a future work, different modifications can be used to improve the performance of the system. Different neural networks and feature set can be used to enhance the performance of the human emotion classification system.

\section{References}

[1] Yoo, Gilsang, SanghyunSeo, Sungdae Hong, and Hyeoncheol Kim (2016) Emotion extraction based on multi bio-signal using back-propagation neural network. Multimedia Tools and Applications, 1-13.

[2] Yin, Zhong, Mengyuan Zhao, Yongxiong Wang, Jingdong Yang, and Jianhua Zhang (2017) Recognition of emotions using multimodal physiological signals and an ensemble deep learning model. Computer methods and programs in biomedicine, 140 , 93-110.

[3] Yanagimoto, Miku, and Chika Sugimoto (2016) Recognition of persisting emotional valence from EEG using convolutional neural networks. Proceedings of IEEE 9th International Workshop on Computational Intelligence and Applications (IWCIA), 27-32.

[4] Xu, Haiyan, and Konstantinos N. Plataniotis (2016) Affective states classification using EEG and semi-supervised deep learning approaches. Proceedings of IEEE 18th International Workshop on Multimedia Signal Processing (MMSP) $1-6$.

[5] Wijeratne, Upani, and UdayangiPerera (2012), Intelligent emotion recognition system using electroencephalography and active shape models. Proceedings of IEEE EMBS Conference on In Biomedical Engineering and Sciences (IECBES), 636-641.

[6] Vijayan, Aravind E, Deepak Sen, and Sudheer (2015) EEG-based emotion recognition using statistical measures and auto-regressive modeling. Proceedings of IEEE International Conference on Computational Intelligence \& Communication Technology (CICT), 587-591.

[7] Ravindran and Malathi.R (2014) Classification of human emotions from EEG signals using filtering and ANFIS classifier. Proceedings of 2nd International Conference on Current Trends in Engineering and Technology (ICCTET), 113-119.

[8] Mohammadpour, Mostafa, Seyyed Mohammad Reza Hashemi, and Negin Houshmand (2017) Classification of EEG-based emotion for BCI applications. Proceedings of IEEE international conference on Artificial Intelligence and Robotics (IRANOPEN), 127-131.

[9] Mohammadi, Zeynab, JavadFrounchi, and Mahmood Amiri (2017) Wavelet-based emotion recognition system using EEG signal. Neural Computing and Applications, 28, no. 8, 1985-1990. 
[10] Lekshmi, S. S., Selvam.V., and Pallikonda Rajasekara (2014) EEG signal classification using principal component analysis and wavelet transform with neural network. Proceedings of IEEE International Conference on Communications and Signal Processing (ICCSP), 687-690.

[11] Ko, Kwang-Eun, Hyun-Chang Yang, and Kwee-Bo Sim (2009) Emotion recognition using EEG signals with relative power values and Bayesian network. International Journal of Control, Automation and Systems, 7, no. 5

[12] Kaundanya, Vaishnavi. L., Anita Patil, and Ashish Panat (2015) Performance of k-NN classifier for emotion detection using EEG signals. Proceedings of IEEE international conference on Communications and Signal Processing (ICCSP), 1160-1164.

[13] Gao, Yongbin, Hyo Jong Lee, and Raja Majid Mehmood (2015) Deep learning of EEG signals for emotion recognition. Proceedings of IEEE international conference on Multimedia \& Expo Workshops (ICMEW), 1-5.

[14] Bhardwaj, Aayush, Ankit Gupta, Pallav Jain, Asha Rani, and Jyoti Yadav (2015) Classification of human emotions from EEG signals using SVM and LDA Classifiers. Proceedings of $2^{\text {nd }}$ International conference on Signal Processing and Integrated Networks (SPIN), 180-185.

[15] Bajaj, Varun, and Ram Bilas Pachori (2014) Human emotion classification from EEG signals using multiwavelet transform. Proceedings of International Conference on Medical Biometrics, 125-130.

[16] Yang, Yimin, QM Jonathan Wu, Wei-Long Zheng, and Bao-Liang Lu (2017) EEG-based emotion recognition using hierarchical network with subnetwork nodes, IEEE Transactions on Cognitive and Developmental Systems.116-125.

[17] Zhang, Jianhai, Ming Chen, Sanqing Hu, Yu Cao, and Robert Kozma (2016) PNN for EEG-based Emotion Recognition. Proceedings of IEEE international conference on Systems, Man, and Cybernetics (SMC), 002319-002323.

[18] Tripathi, Samarth, Shrinivas Acharya, Ranti Dev Sharma (2017) Sudhanshu Mittal, and Samit Bhattacharya (2017) Using Deep and Convolutional Neural Networks for Accurate Emotion Classification on DEAP Dataset. Proceedings of International Conference on AAAI, 4746-4752.

[19] Li, Jinpeng, Zhaoxiang Zhang, and Huiguang He (2017) Hierarchical Convolutional Neural Networks for EEG-Based Emotion Recognition. Cognitive Computation , 1-13.

[20] Thejaswini, S., KM Ravi Kumar, Shyam Rupali, and Vijayendra Abijith (2018) EEG Based Emotion Recognition Using Wavelets and Neural Networks Classifier. Proceedings of International conference on Cognitive Science and Artificial Intelligence (Springer), 101-112.

[21] Mahajan and Rashima (2018) Emotion Recognition via EEG Using Neural Network Classifier. Soft Computing: Theories and Applications - Springer, 429-438.

[22] Tang, Hao, Wei Liu, Wei-Long Zheng, and Bao-Liang Lu (2017) Multimodal Emotion Recognition Using Deep Neural Networks. Proceedings of International Conference on Neural Information Processing (Springer). 811-819.

[23] Lin, Yuan-Pin, Chi-Hong Wang, Tzyy-Ping Jung, Tien-Lin Wu, Shyh-Kang Jeng, Jeng-Ren Duann, and Jyh-Horng Chen. (2010) EEG-based emotion recognition in music listening. IEEE Transactions on Biomedical 
Engineering , 57, no. 7, 1798-1806.

[24] Anh, Viet Hoang, Manh Ngo Van, Bang Ban Ha, and Thang Huynh Quyet (2012) A real-time model based support vector machine for emotion recognition through EEG. Proceedings of International conference on Control, Automation and Information Sciences (ICCAIS), 191-196.

[25] Pouyanfar, Samira, and Hossein Sameti (2014) Music emotion recognition using two level classification. Proceedings of IEEE international conference on Intelligent Systems (ICIS), 1-6.

[26] S. Koelstra, C. Muehl, M. Soleymani, J.-S. Lee, A. Yazdani, T. Ebrahimi, T. Pun, A. Nijholt, I. Patras (2012) IEEE Transaction on Affective Computing, vol.3, no.1, pp: 18-31.

[27] D. Jude Hemanth, C Kezi Selva Vijila, A Immanuel Selvakumar and J Anitha (2014) Neurocomputing vol.130, pp: 98-107.

[28] Marcus Santos et al. Online heart monitoring systems on the internet of health things environments: A survey, a reference model and an outlook. Information Fusion, v. 53, p. 222-239, 2020.

[29] Marinho Leandro et al. A novel electrocardiogram feature extraction approach for cardiac arrhythmia classification. Future Generation Computer Systems, v. 97, p. 564-577, 2019.

[30] Jardes Rodrigues et al. Classification of EEG Signals to Detect Alcoholism Using Machine Learning Techniques. PATTERN RECOGNITION LETTERS, v. 125, p. 140-149, 2019.

[31] Luis Periera et al. Automatic identification of epileptic EEG signals through binary magnetic optimization algorithms. NEURAL COMPUTING \& APPLICATIONS (INTERNET), v. 31, p. 1317-1329, 2019.

[32] Moraes JL et al. Advances in Photopletysmography Signal Analysis for Biomedical Applications. SENSORS, v. 18, p. 1894-1920, 2018.

[33] Enas Abdulhay et al. Computer-aided autism diagnosis via second-order difference plot area applied to EEG empirical mode decomposition. NEURAL COMPUTING \& APPLICATIONS, v. 1, p. 1-10, 2018.

[34] Roberto Munoz et al. A new EEG software that supports emotion recognition by using an autonomous approach. NEURAL COMPUTING \& APPLICATIONS, v. 1, p. 1-17, 2018. 